\title{
Psychological, pharmacological, and combined treatments for binge eating disorder: A systematic review and meta-analysis
}

\author{
Ata Ghaderi Corresp., 1 , Jenny Odeberg ${ }^{2}$, Sanna Gustafsson ${ }^{3,4}$, Maria Råstam ${ }^{5,6}$, Agneta Brolund ${ }^{2}$, Agneta \\ Pettersson ${ }^{2}$, Thomas Parling ${ }^{4,7}$ \\ 1 Division of Psychology, Department of Clinical Neuroscience, Karolinska Institute, Sweden \\ 2 Swedish Agency for Health Technology Assessment, and Assessment of Social Services, Stockholm, Sweden \\ 3 University Health Care Research Centre, Faculty of Medical Sciences, University College of Örebro, Örebro, Sweden \\ ${ }^{4}$ Centre for Psychiatry Research, Department of Clinical Neuroscience, Karolinska Institute, Stockholm, Sweden \\ 5 Department of Clinical Sciences, Lund University, Lund, Sweden \\ 6 Gillberg Neuropsychiatry Centre, University of Gothenburg, Gothenburg, Sweden \\ 7 Stockholm Health Care Services, Stockholm County Council, Stockholm, Sweden \\ Corresponding Author: Ata Ghaderi \\ Email address: ata.ghaderi@ki.se
}

Objective: To systematically review the efficacy of psychological, pharmacological and combined treatments for binge eating disorder (BED). Method: Systematic search and meta-analysis. Results: We found 45 unique studies with low/medium risk of bias, and moderate support for the efficacy of Cognitive Behavior Therapy (CBT) and CBT guided self-help (with moderate quality of evidence), and modest support for Interpersonal Psychotherapy (IPT), Selective Serotonin Reuptake Inhibitors (SSRI) and lisdexamfetamine (with low quality of evidence) in the treatment of adults with BED in terms of cessation of or reduction in the frequency of binge eating. The results on weight loss were disappointing. Only lisdexamfetamine showed a very modest effect on weight loss (low quality of evidence). While there is limited support for the long-term effect of psychological treatments, we have currently no data to ascertain the long-term effect of drug treatments. Some undesired side effects are more common in drug treatment compared to placebo, while the side effects of psychological treatments are unknown. Direct comparisons between pharmaceutical and psychological treatments are lacking as well as data to generalize these results to adolescents. Conclusions: We found moderate support for the efficacy of CBT and guided self-help for the treatment of BED. However, IPT, SSRI and lisdexamfetamine received only modest support in terms of cessation of or reduction in the frequency of binge eating. The lack of long-term follow-ups is alarming, especially with regard to medication. Long-term follow-ups, standardized assessments including measures of quality of life, and the study of underrepresented populations should be a priority for future research. 
1 Psychological, pharmacological, and combined treatments for binge eating disorder: A

2

\author{
systematic review and meta-analysis
}

\section{Running title:}

\section{Systematic review of treatment of BED} MD 6,7, Agneta Brolund ${ }^{2}$, Agneta Petterson, $\mathrm{PhD}^{2}$, Thomas Parling, $\mathrm{PhD}{ }^{4,5}$

\footnotetext{
${ }^{1}$ Division of Psychology, Department of Clinical Neuroscience, Karolinska Institutet, Stockholm, Sweden (ata.ghaderi@ki.se)

2 Swedish Agency for Health Technology Assessment, and Assessment of Social Services, Stockholm, Sweden (Jenny.Odeberg@sbu.se: Agneta.Brolund@sbu.se:

Agneta.Pettersson@sbu.se)

${ }^{3}$ University Health Care Research Center, Faculty of Medicine and Health, Örebro University, Sweden (sanna.aila-gustafsson@regionorebrolan.se)

${ }^{4}$ Centre for Psychiatry Research, Department of Clinical Neuroscience, Karolinska Institutet

${ }^{5}$ Stockholm Health Care Services, Stockholm County Council, Stockholm, Sweden (Thomas.parling@ki.se)

${ }^{6}$ Department of Clinical Sciences Lund, Lund University, Lund, Sweden (maria.rastam@med.lu.se)

${ }^{7}$ Gillberg Neuropsychiatry Centre, University of Gothenburg, Göteborg, Sweden
}

\section{Corresponding author:}

Professor Ata Ghaderi

Division of Psychology, Department of Clinical Neuroscience, Karolinska Institutet

SE 17177, Stockholm, Sweden

E-mail address: ata.ghderi@,ki.se

ORCID ID: orcid.org/0000-0001-8483-7964 
Objective: To systematically review the efficacy of psychological, pharmacological and combined treatments for binge eating disorder (BED). Method: Systematic search and meta-analysis. Results: We found 45 unique studies with low/medium risk of bias, and moderate support for the efficacy of Cognitive Behavior Therapy (CBT) and CBT guided self-help (with moderate quality of evidence), and modest support for Interpersonal Psychotherapy (IPT), Selective Serotonin Reuptake Inhibitors (SSRI) and lisdexamfetamine (with low quality of evidence) in the treatment of adults with BED in terms of cessation of or reduction in the frequency of binge eating. The results on weight loss were disappointing. Only lisdexamfetamine showed a very modest effect on weight loss (low quality of evidence). While there is limited support for the long-term effect of psychological treatments, we have currently no data to ascertain the long-term effect of drug treatments. Some undesired side effects are more common in drug treatment compared to placebo, while the side effects of psychological treatments are unknown. Direct comparisons between pharmaceutical and psychological treatments are lacking as well as data to generalize these results to adolescents. Conclusions: We found moderate support for the efficacy of CBT and guided self-help for the treatment of BED. However, IPT, SSRI and lisdexamfetamine received only modest support in terms of cessation of or reduction in the frequency of binge eating. The lack of longterm follow-ups is alarming, especially with regard to medication. Long-term follow-ups, standardized assessments including measures of quality of life, and the study of underrepresented populations should be a priority for future research. 
58

59

60

61

62

63

64

65

66

67

68

69

70

\section{Introduction}

Binge eating disorder (BED) became a formal diagnosis within the Eating and Feeding disorders in the fifth edition of the Diagnostic and statistical Manual for Mental Disorders (DSM-5: American Psychiatric Association 2013). BED is characterized by episodes of binge eating defined as eating, in a discrete period of time, an amount of food that is definitely larger than most people would eat during similar circumstances while experiencing a lack of control over eating.

Epidemiological studies suggest that 1-4\% in the population suffer from BED (Hoek 2006;

Kessler et al. 2013). The onset is usually in the late adolescent or early adult years (Nicholls et al. 2011) with an increasing incidence in the late adolescence and early adulthood in both sexes (Hudson et al. 2007; Stice et al. 2013). Recent studies suggest that the lifetime prevalence of BED (1.4\%) is higher than that of bulimia nervosa $(0.8 \%)($ Kessler et al. 2013).

Patients with BED often present with comorbid psychiatric and somatic diagnoses, lower quality of life, more suicide ideation and attempts, and lower social functioning compared to the general population (Agh et al. 2015; Favaro \& Santonastaso 1997; Grilo et al. 2009; Hudson et al. 2007; Kessler et al. 2013; Ulfvebrand et al. 2015; Wilfley et al. 2003). In studies using semi-structured interviews, the lifetime prevalence of obesity in BED-patients is 42-49\% (Hudson et al. 2007; Kessler et al. 2013), which translates into an increased risk of conditions as diabetes and metabolic syndrome (Citrome 2017), with increased health care consumption and health care costs (Agh et al. 2015).

In terms of treatment interventions, Cognitive Behavior Therapy (CBT), CBT guided self-help (CBT-gsh), Interpersonal Psychotherapy (IPT), behavior weight loss (BWL), dialectical behavior therapy (DBT), pharmaceutical treatments and different combinations of medical and psychological treatments have been studied (e.g., Fairburn et al. 2015; Grilo 2017; McElroy 2017). CBT and CBT-gsh are the most well studied treatments, with a large number of studies. BWL programs have been evaluated in individual (Wilson 2011), group (Munsch et al. 2007), and self-help program formats (Grilo \& Masheb 2005). Since a majority of BED patients also suffer from overweight or obesity many treatments have not only addressed the binge-eating behavior, but also aimed at weight loss (e.g., Agras et al. 1994; Grilo 2016). 
84 Some studies have also investigated the effect of bariatric surgery on binge eating behaviors, in addition

85 to its weight loss effects (Colles et al. 2008; de Man Lapidoth et al. 2011; de Zwaan et al. 2010; McElroy 86 et al. 2011a; McElroy et al. 2011b).

87 In terms of pharmacological treatments, the efficacy of a broad spectrum of

88 pharmacotherapeutic agents on binge eating frequency and weight loss have been investigated (McElroy

89 2017). Given the short-term efficacy of anti-depressants, specifically fluoxetine, for bulimia nervosa, anti-

90 depressants for BED has been studied in several randomized controlled trials (RCT). Due to high

91 comorbidity of overweight and obesity in BED (de Man Lapidoth et al. 2006; de Zwaan 2001; Decaluwe

92 \& Braet 2003; Vamado et al. 1997), the efficacy of anti-obesity agents such as fenfluramine, orlistat and

93 sibutramine have also been investigated. On the basis of the hypothesized biological underpinnings of

94 BED or similarity with some other conditions such as addiction disorders (McElroy 2017), other drugs

95 such as antiepileptics (e.g., topiramate, and lamotrigine) as well as drugs that are usually prescribed for

96 attention deficit and hyperactivity disorders (e.g., lisdexamfetamine), or anti-addiction drugs (e.g.,

97 naloxone) have also been tested with binge eating frequency and weight loss as main outcome variables.

98 Most of the pharmacological trials are short-term interventions (6-16 weeks) with effects investigated at

99 post-treatment, but almost no long-term follow-ups exist.

100 Several previous reviews and meta-analyses of the treatment of BED have focused on RCTs

101 (e.g., Amianto et al. 2015; Brownley et al. 2016; Brownley et al. 2015; Grilo 2017; Iacovino et al. 2012;

102 McElroy 2017; McElroy et al. 2015b; Reas \& Grilo 2014; Reas \& Grilo 2015; Vocks et al. 2010). Nine

103 RCTs (Brownley et al. 2016) investigated psychological treatments versus waitlist controls and 25 RCTs

104 investigating the effect of drugs. Significantly more participants achieved abstinence from binge eating

105 with CBT versus waitlist. Other forms of psychological treatments such as dialectic behavior therapy and

106 behavioral weight loss also reduced binge-eating and related psychopathology. CBT (whether delivered in

107 therapist-led, partially therapist-led, or CBT-gsh) did not significantly reduce weight or symptoms of

108 depression. Interestingly, the authors defined many forms of therapy such as psychodynamic

109 psychotherapy as a BED-focused CBT. Further, the review reported that second-generation 
110 antidepressants (SGA) such as citalopram, fluoxetine, and sertraline decreased binge eating and reduced

111 symptoms of depression. Similar outcomes were found for topiramate as well. Lisdexamfetamine reduced

112 binge-eating and binge-eating-related obsessions and compulsions. Only lisdexamfetamine and

113 topiramate reduced weight. However, the authors note the inconsistencies of outcome measures across

114 trials and the paucity of assessments beyond the end of treatment (Brownley et al. 2015). A review

115 evaluating controlled studies of pharmacotherapy for BED found 22 RCTs (Reas \& Grilo 2015) of which

11614 were pharmacotherapy-only trials and 8 trials investigating combinations with CBT and/or BWL. All

117 but two studies had been reported in an earlier report by the authors (Reas \& Grilo 2014). Reviews of

118 RCTs suggest that the outcome of psychological treatments, alone or in combination with drugs, are

119 superior to drugs only, while the combined treatments of BED (Grilo 2017; Reas \& Grilo 2014) failed to

120 show superiority compared to CBT only. Nevertheless, adding some drugs to psychological treatments

121 might enhance the level of weight loss, compared to CBT or BWL treatment only, although the effects are

122 modest (Reas \& Grilo 2014). Summing up, reviews of treatment research of BED showed that CBT,

123 individually or in groups, was most researched and produced the best results in terms of proportion of

124 patients reaching remission compared to waiting list. The evidence-base for pharmacotherapy was

125 limited. In the short term SGAs seem to reduce the number of binge episodes but there are almost no

126 RCTs on its longer-term efficacy.

\section{Aims of the study}

128 Given the importance of replications, the aim of the present study was to evaluate the efficacy

129 and quality of evidence, as well as potential iatrogenic effects of treatment in controlled psychological,

130 pharmacological, and combined treatment interventions for BED. Outcomes of interest were remission,

131 episodes of binge eating, weight loss, measures of specific psychopathology of eating disorders,

132 depressive symptoms, quality of life, and side effects. We included only studies characterized by low or

133 moderate risk of bias. 
136 The systematic review was conducted in accordance with the PRISMA statement (Moher et al. 2009).

137 The inclusion criteria were as follows: randomized controlled trials (RCT) and prospective controlled

138 clinical trials (CCT), participants with full or threshold BED according to DSM-IV (American Psychiatric

139 Association 2000) research criteria, or BED according to DSM-5 (American Psychiatric Association

140 2013) regardless of age or weight status, and all types of interventions except for outdated treatments (i.e.,

141 studies investigating drugs that have been withdrawn from the market due to serious side effects).

142 Outcomes of interest were remission, episodes of binge eating, weight loss, measures of specific

143 psychopathology of eating disorders, depressive symptoms, quality of life, and side effects. We did not

144 decide upon imposing any a priori specific inclusion or exclusion criteria regarding the duration of

145 treatment, length of follow-up(s) or number of participants.

\section{Search strategy}

The databases PubMed (NLM), Embase (Elsevier), Cochrane Library (Wiley), Scopus and HTA databases from Centre for reviews and disseminations (CRD) were searched until November 2015. The search was updated again in November 2016. Reference lists and books were also used to identify further studies. Search strategies are listed in Appendix A. Two reviewers screened the titles and abstracts independently. Full text articles were retrieved if one or both reviewers considered a study potentially

152 eligible. Both reviewers read the full texts, and consensus was reached regarding eligibility. The excluded 153 articles are provided in Appendix B.

154 Two pairs of reviewers assessed eligible studies for risk of bias independently. Studies were 155 scored as having either high or acceptable (i.e., low or medium) risk of bias. Assessment of the risk of 156 bias of each study was based on the quality of the randomization procedure and equality of the conditions 157 before the treatment, allocation concealment, blinding (participants, assessors, treatment providers), dropout $(<30 \%$ of total sample and $<10 \%$ difference between the conditions), potential conflict of interest, and analysis of confounders. Studies were excluded if they were not controlled, or if the drop-out rate was lager than those reported above. In addition to independent ratings by at least two experts for each paper, 
161 whenever any minor issues related to randomization, allocation, blinding etc. were unclear, they were

162 thoroughly discussed by the entire group to reach consensus on the level of risk of bias. Only studies with

163 acceptable risk of bias were included in this review.

\section{Data management}

165 For all studies, we extracted country, type of setting, method of recruitment, type of treatment,

166 treatment length, number of sessions, treatment format, age, Body Mass Index (BMI) and gender.

167 Extraction from drug treatments also included dosage during the trial. Study characteristics are reported in

168 Appendix C. Outcomes included remission defined as complete cessation of binge eating, BE frequency

169 (i.e. number of binges/week), BMI (weight reduction), specific psychopathology of eating disorders

170 measured by specific measures such as the Eating Disorder Examination Questionnaire (Fairburn, 2008),

171 depressive symptoms, and quality of life, and side effects.

\section{Statistical analysis}

173 For dichotomous outcomes, we estimated the risk difference (RD) or risk ratio (RR) and for

174 continuous outcomes we estimated the mean difference (MD) or standard mean difference (SMD). All

175 outcomes were reported with $95 \%$ confidence intervals (CI). Dichotomous effects were weighted using

176 the Mantel Haenszel method and continuous effects were weighted using Inverse Variance.

177 In one case we contacted the authors and received supplementary data from one study (Schlup

178 et al. 2009), which enabled inclusion in the meta-analysis. Data synthesis was carried out using Rev Man

179 (5.3) (Review Manager (RevMan) 2014), employing the random effects model due to clinical

180 heterogeneity.

181 The certainty of evidence was assessed with Grading of Recommendations Assessment, Development and

182 Evaluation (GRADE: strong, moderate, low or insufficient) (Guyatt et al. 2008). In brief, preliminary

183 certainty of the evidence was classified as high (labeled $\oplus \oplus \oplus \oplus$ ) if the results were based on data from

184 RCT, otherwise the preliminary certainty of evidence starts with low (labeled $\oplus \oplus \mathrm{OO}$ ). Thereafter, we 185 analyzed to what extent the results from the meta-analysis might be affected by the five risk domains in 
186 GRADE. These are: overall risk of bias across studies, degree of heterogeneity between studies

187 (inconsistency), size of the confidence intervals for the summary measures (imprecision), risk for

188 publication bias and risk that the results are not generalizable to the actual context (indirectness). The

189 final certainty of the evidence depended on whether there were severe deficiencies in any of the five risk

190 domains. Thus, the resulting certainty of evidence could be high $(\oplus \oplus \oplus \oplus)$, moderate $(\oplus \oplus \oplus \mathrm{O})$, low

$191(\oplus \oplus \square \square)$ or insufficient/very low $(\oplus \oplus \mathrm{OO})$. One special rule was applied; if an intervention was

192 evaluated in only one small study $($ size $<100)$ the certainty of the evidence was assessed as very

193 low/insufficient $(\oplus \mathrm{OOO})$.

194

195

196

197

198

199

200

201

202

203

204

205

206

\section{7}

208

209

210

\section{Results}

The search of the databases resulted in 3595 publications and the abstracts of these were screened (See Figure 1). Of these, 296 were obtained in full text and screened. A total of 99 were then classified as relevant according to the inclusion and exclusion criteria. At this stage we classified the trials according to drug, psychotherapy, or a combination of drug and psychotherapy (1:st classification). Among the selected 99 publications we found 45 unique studies (54 publications) with low/medium (i.e., acceptable) bias risk. A total of 45 publications were assessed as high bias risk and were not included in the analysis. See Appendix B for excluded publications.

Please insert Figure 1 here

Taken together, the most common reasons for exclusion due to high risk of bias were unclear randomization, allocation, whether assessors were blinded and high drop out.

\section{Study characteristics}

All of the included studies were RCT's. The studies included 4611 participants (range 18-773) and they were conducted in North America and Europe with the exception of one study from South America. Participants were 18 years and older, recruited mainly from out-patient settings and through direct 
211 advertisement to the community, and the majority were women. Standardized diagnostic interviews were

212 used to establish BED diagnosis. The majority of studies had a lower inclusion criteria regarding BMI in

213 the overweight to obese weight spectrum. However, the outcome of the treatment of BED was not

214 presented separately for participants with or without concurrent obesity in any of the studies. Please see

215 Appendix C for detailed characteristics of included studies.

\section{Interventions}

217 The trials included a variety of interventions and various combinations of them (drugs,

218 psychotherapy, behavioral weight loss (BWL), and low-energy-density diet counseling) in different

219 formats (individual or group, as well as self-help with or without support) and different control conditions

220 (wait list or placebo). We first classified the trials according to drug, psychotherapy, or a combination of

221 drug and psychotherapy (1:st classification). In the second step, we identified and matched those with

222 similar treatment and control condition. This resulted in the following potential meta-analyses: Drugs vs.

223 placebo (SSRI, lisdexamfetamine, mood stabilizer, anorexiants), combination of drug and CBT/CBT-gsh

224 vs. placebo, CBT vs. wait list, CBT-gsh vs. wait list, IPT vs. CBT/CBT-gsh, and BWL vs. CBT/CBT-gsh.

\section{Included studies not in the meta-analyses per 1:st classification}

Drug vs. placebo: For some of the remaining studies with acceptable risk of bias, only one study

per drug was identified. There was insufficient number of studies to assess the efficacy of the following dulextin (Serotonin-norepinephrine reuptake inhibitor; SNRI)(Guerdjikova et al. 2012), baclofen (muscle relaxant)(Corwin et al. 2012), imipramine (tricyclic antidepressant) (Laederach-Hofmann et al. 1999) and armodafinil (sympathomimetic) (McElroy et al. 2015a). One study investigated fluoxetine vs. sertraline

232 (Leombruni et al. 2008). Thus, a total of 14 studies were included in the meta-analyses for drugs vs.

233 placebo for BED.

234 Combination of drug and psychological treatment: The remaining five studies with acceptable

235 risk of bias investigated unique combinations of drug and psychological treatment. All studies were too 236 small $(\mathrm{n}<100)$ and thus there was low certainty of the evidence to assess the following combinations; 
237 desipramine (tricyclic anti-depressive) + CBT (Agras et al. 1994), topiramate (anticonvulsive) combined 238 with CBT (Claudino et al. 2007), orlistat (anorexiant) combined with CBT-gsh (Grilo et al. 2005a),

239 orlistat (anorexiant) combined with BWL (Grilo \& White 2013), and fluoxetine (SSRI) combined with 240 CBT (Grilo et al. 2005b).

241 Psychological treatment: Of the remaining 24 studies (i.e., 31 publications) with acceptable risk 242 of bias, 13 were not included in any meta-analysis. Alfonsson (2015) investigated behavioral activation 243 vs. wait list, and Shapiro (2007) investigated CBT for healthy eating and weight control in group format 244 as one of three arms (the wait list vs. CBT-gsh comparison were included in the CBT-gsh vs. wait list 245 meta-analyses). These two interventions were assessed as providing a too general CBT approach not 246 including the specific eating disorder interventions as the included publications in the meta-analysis for 247 CBT vs. wait list. The mindfulness based eating awareness training condition and psychoeducation with elements of CBT condition vs. wait list (Kristeller et al. 2014) were excluded due to too low similarity with the included interventions in the meta-analysis for CBT vs. wait list. In addition, in some studies only one study per intervention was identified; brief strategic therapy vs. CBT (Castelnuovo et al. 2011), adapted motivational interviewing combined with self-help vs. self-help (Cassin et al. 2008), self-help combined with Treatment as Usual (TAU) vs. TAU (Grilo et al. 2013), shape exposure CBT vs. cognitive restructuring CBT (Hilbert \& Tuschen-Caffier 2004), CBT combined with low calorie diet (Masheb et al. 2011), CBT vs. CBT in group format (Ricca et al. 2010) and finally a study with three conditions; CBT vs. schema therapy vs. Appetite focused CBT (McIntosh et al. 2016).

In Carter (1998), only the CBT-gsh vs. wait list was included (the third condition, self-help, was not included in any meta-analysis) (Carter \& Fairburn 1998). The CBT vs. BWL comparison from the Grilo (2011) study was included while the third arm (CBT+BWL) was not included in any meta-analysis.

259 In Kelly et al (2014) the self-help meal planning combined with self-compassion therapy arm was not 260 included. However, we included the CBT-gsh vs. wait list comparisons (Kelly \& Carter 2014).

\section{Overall evidence quality}


263 most common reasons for downgrading were precision and study quality. Only four meta-analyses

264 received moderate rating (the highest level of quality in this review assigned to any single study) and the 265 majority received limited evidence of quality. Please see Table 1 for details.

\section{Meta-analyses}

In the following, we present the summary of the meta-analyses (please see Table 1 for detailed

268

269

270

271

272

273

274

275

276

277

278

279

280

281

282

283 284 2005b).

285

286

287

Please insert Table 1 here

\section{SSRI vs. placebo}

SSRI vs. placebo was investigated in six studies (Grilo et al. 2012; Grilo et al. 2005b; Guerdjikova et al. 2008; Hudson et al. 1998; McElroy et al. 2000; McElroy et al. 2003; Pearlstein et al. 2003).

Participants' age were 18-60 years, mostly women ( $>70 \%$ ) with weight $>85 \%$ of ideal body weight.

Treatments ranged between 6-16 weeks and only one study reported 6 and 12 months follow ups (Grilo et al. 2012; Grilo et al. 2005b). Flexible dosage and titration was used in all studies but one (Grilo et al.

Remission was reported in all six studies $(n=264)$. The $\mathrm{RD}=0.15$ was in favor of SSRI treatment compared with placebo at the end of the treatment. Binge eating frequency was reported in all six studies $(n=257)$, and comparisons resulted in SMD $=-0.45$ in favor of SSRI treatment compared with 
288 placebo at the end of the treatment. Eating disorder psychopathology was only reported in one study

289 comparing SSRI vs. placebo (Grilo et al. 2005b). BMI was reported in five studies $(n=237$ ) (Grilo et al.

290 2005b; Guerdjikova et al. 2008; Hudson et al. 1998; McElroy et al. 2000; McElroy et al. 2003). There

291 was no significant difference for SSRI vs. placebo. Symptoms of depression were reported in four studies

$292(n=148)$ (Grilo et al. 2005b; Guerdjikova et al. 2008; McElroy et al. 2003; Pearlstein et al. 2003)

293 showing no significant difference between SSRI and placebo.

294 Side effects. Hudson et al reported significantly more insomnia, nausea, and abnormal dreams in

295 the fluoxetine condition compared with placebo (Hudson et al. 1998). McElroy et al (2003) reported

296 significantly more fatigue and perspiration in the citalopram group compared with the placebo group

297 (McElroy et al. 2003). There were significantly more participants in the sertraline group who reported

298 insomnia (McElroy et al. 2000). One study reported 1.3 to 4 times more sedation, nausea, dry mouth and

299 decreased libido (Pearlstein et al. 2003).

$300 \quad$ Lisdexamfetamine vs. placebo

301 One research group had two publications $(n=850)$ (McElroy et al. 2015c; McElroy et al. 2015d)

302 including a multicenter study with participants from Holland, Spain and Sweden (McElroy et al. 2015c).

303 Hence, the results from three samples are combined for this meta-analysis. Participants were 18-55 years,

304 mostly women $>81 \%$ with a BMI range of $18-45$. Treatment length was 11 or 12 weeks. One study

305 (McElroy et al. 2015d) investigated the effect of flexible dosages (30-70 mg/day), while the other one

306 (Guerdjikova et al. 2016) administered a standardized dosage (70 mg/day). The outcomes are based on

307 those who received $70 \mathrm{mg} /$ day. The studies did not report any follow up data.

308 Remission was reported in three studies $(n=850)$. The $\mathrm{RD}=0.25$ was in favor of lisdexamfetamine

309 compared to those receiving placebo at the end of treatment. Binge eating frequency was reported in all

310 three studies $(n=849)$. There was a significant difference in favor for lisdexamfetamine compared with

311 placebo (Table 1). Eating disorder psychopathology was not reported. BMI: Three studies reported

312 weight difference from baseline $(n=852)$, and the result ( $\mathrm{SMD}=-5.23)$ favored the lisdexamfetamine 
313 condition compared with placebo. Symptoms of depression were reported from one study only (McElroy 314 et al. 2015c).

315 Side effects. In both publications, a majority of participants in the lisdexamfetamine group ( $>80 \%)$ 316 and the placebo group ( $>50 \%$ ) reported side effects. In the placebo group, 0-3\% discontinued due to side

317 effects compared with $3-6 \%$ in the lisdexamfetamine group. One participant died due to

318 methamphetamine and amphetamine toxicity (McElroy et al. 2015d).

319

\section{Anti-convulsive vs. placebo}

Two studies investigated the effect of anti-convulsive medication for their mood stabilization

322

323

324

325

326

327

328

329

330

331

332

333

334

335

336

337 characteristics vs. placebo $(n=443)$ (Guerdjikova et al. 2009; McElroy et al. 2007). Participants were between 18-65 years with $\mathrm{BMI}>30($ mean $\mathrm{BMI}>38.5)$ and the majority were women $(>80 \%)$. The treatment in both studies was 16 weeks with no follow-ups. Both studies used flexible dosage of lamotrigin (Guerdjikova et al. 2009) and topiramate (Guerdjikova et al. 2009; McElroy et al. 2007).

Remission: The outcome in terms of remission was inconsistent between the two studies $(n=443)$, resulting in a non-significant outcome. Binge eating frequency was inconsistent $(n=445)$ between the two studies, resulting in a non-significant outcome (Table 1). Eating disorder psychopathology was only reported in one study (Guerdjikova et al. 2009). BMI: only one study reported data in a proper format for inclusion in a meta-analysis (McElroy et al. 2007). Symptoms of depression were investigated in both studies but only one study reported the mean and thus, no meta-analysis was performed.

Side effects. In the topiramate study (McElroy et al. 2007), significantly more participants in the intervention condition compared to placebo reported the following adverse events: paresthesia, upper respiratory tract infection, taste perversion, difficulty with concentration/attention, and difficulty with memory not otherwise specified (McElroy et al. 2007). 
339 (800-1000 $\mu \mathrm{g} /$ day) (Brownley et al. 2013) and Orlistat (120 mg/day) (Golay et al. 2005). Participants

340 were 18-65 years (mean ages were 37 and 41 ) and they were mostly women ( $>83 \%)$ with a mean BMI of

34137 and 43 respectively. One study included overweight and obese (Brownley et al. 2013) while the other

342 included only obese participants. The duration of the treatments were six months in both studies with no

343 follow-ups.

The outcome measures were presented in different formats between the two studies such that no meta-analysis was performed. Remission: Only one study reported results on remission (Golay et al. 2005). There was no significant difference between the treatment and the placebo group. Binge eating

347 frequency was reported in both studies but with no significant differences between treatment and placebo 348 in both studies. Eating disorder psychopathology was reported in one study (Brownley et al. 2013) with no significant differences between treatment groups and placebo. BMI: weight reduction was significantly larger for the orlistat group compared with placebo (Golay et al. 2005). Symptoms of depression: there were no significant effects in either study. Quality of life was presented in one study with no significant effect (Golay et al. 2005).

Side effects. No significant differences between groups were reported in the chromium picolinate study (Brownley et al. 2013) and no side effects were reported in the orlistat study (Golay et al. 2005).

\section{Drug \& psychological treatment combined}

The five studies could not be combined for a meta-analysis (Agras et al. 1994; Claudino et al. 2007; Grilo et al. 2005a; Grilo et al. 2005b; Grilo \& White 2013). The following combinations were investigated: A combination of desipramine, weight loss, and CBT vs. CBT combined with weight loss program (Agras et al. 1994); topiramate combined with CBT versus CBT (Claudino et al. 2007); combination of orlistat, dietary advice, and CBT vs. placebo, dietary advice, and CBT (Grilo et al. 2005a); a combination of fluoxetine and CBT vs. placebo and CBT (Grilo et al. 2005b); and a 
364 years old, $>78 \%$ were women, with $\mathrm{BMI} \geq 27$. Treatments were 12 to 36 weeks and followed up at three

365 months (Agras et al. 1994; Grilo et al. 2005a), 6 months (Grilo \& White 2013), and at 12 months (Grilo et 366 al. 2012).

367 Side effects. Two studies did not report on side effects (Agras et al. 1994; Grilo et al. 2005b).

368 Those who received topiramate reported significantly more paresthesia, taste perversion, dysuria, and leg

369 pain compared with the placebo group. However, the placebo group reported significantly more insomnia

370 (Claudino et al. 2007). Those who received orlistat reported more gastrointestinal events that resolved

371 (Grilo et al. 2005a; Grilo \& White 2013). However, two participants dropped out due to these side effects

372 (Grilo et al. 2005a).

373

374

\section{CBT vs. wait list}

375

CBT vs. wait list was investigated in four studies(Dingemans et al. 2007; Grilo et al. 2005b;

376

377

378

379

380

381

382

383

384

385

386

387

388

389

Peterson et al. 2009; Schlup et al. 2009). Participants were mostly women (>70\%), 18-65 years old, with a mean age of 43.5 years and a mean BMI of 36.6. Treatments ranged between 8 (Schlup et al. 2009), 16 (Grilo et al. 2005b), and 20 weeks (Dingemans et al. 2007; Peterson et al. 2009), and the number of sessions ranged between 8 and 16. No follow up data were presented. The effects were investigated in individual format in one study (Grilo et al. 2005b), while the other three studies used group format (Dingemans et al. 2007; Peterson et al. 2009; Schlup et al. 2009). The content of the interventions was assessed as fairly equal despite different formats, number of sessions and the duration of treatment. Remission: Four studies reported remission $(n=272)$ in favor of CBT compared with wait list, $\mathrm{RD}=0.40$. Binge eating frequency was reported in all four studies $(n=272)$, however due to different frequency assessments (BE episodes (Grilo et al. 2005b; Schlup et al. 2009), and BE days during the last 28 days (Dingemans et al. 2007; Peterson et al. 2009)) the results in the meta-analysis are presented as $\mathrm{SMD}$. The results were in favor of CBT compared with wait list, $\mathrm{SMD}=-0.83$. Eating disorder psychopathology was investigated with the EDE-Q and presented in four studies $(n=269)$ (Dingemans et al. 2007; Grilo et al. 2005b; Peterson et al. 2009; Schlup et al. 2009). The results favor the CBT 
390 intervention compared with the wait list, $\mathrm{SMD}=-0.50 . B M I$ : Three of the four studies reported data for

391 inclusion in the meta-analysis $(n=220)$ on BMI change (Grilo et al. 2005b; Peterson et al. 2009; Schlup

392 et al. 2009). No significant effect was found (Table 1). The fourth study did not show any significant

393 results either (Dingemans et al. 2007). Symptoms of depression were reported in four studies $(n=267)$

394 (Dingemans et al. 2007; Grilo et al. 2005b; Peterson et al. 2009; Schlup et al. 2009). Different outcome

395 scales were used in the studies but were synthesized for this meta-analysis showing a result in favor of the

396 CBT compared with the wait list condition, $\mathrm{SMD}=-0.42$.

397 Side effects were not reported in any of the studies.

398

399

400

401

402

403

404

405

406

407

408

409

410

411

412

413

414

415

\section{CBT self-help vs. wait list}

There were eight studies investigating CBT-gsh vs. wait list(Carrard et al. 2011; Carter \&

Fairburn 1998; Grilo \& Masheb 2005; Grilo et al. 2014; Kelly \& Carter 2014; Masson et al. 2013;

Shapiro et al. 2007; ter Huurne et al. 2015). Participants were 18 years and older and 87\% were women.

Two studies included only overweight / obese participants (BMI $\geq 27)$ (Grilo \& Masheb 2005; Shapiro et al. 2007) and one study included only obese participants (Grilo et al. 2014). The remaining studies had no BMI inclusion criteria. The number of treatment weeks varied between 3 (Kelly \& Carter 2014), 10-13 (Carter \& Fairburn 1998; Grilo \& Masheb 2005; Masson et al. 2013; Shapiro et al. 2007), 15-18 (ter Huurne et al. 2015), 16 (Grilo et al. 2014), and 24 (Carrard et al. 2011). There were five studies (Carrard et al. 2011; Carter \& Fairburn 1998; Grilo \& Masheb 2005; Grilo et al. 2014; ter Huurne et al. 2015) that investigated eating disorder specific CBT, one study investigated DBT (Masson et al. 2013) and one study investigated a treatment with focus on healthy eating and weight control with CBT)(Shapiro et al. 2007). The degree of support varied between the studies: it ranged between no support (Carter \& Fairburn 1998; Grilo et al. 2014), only one session before the program started (Kelly \& Carter 2014), one phone call (Shapiro et al. 2007), 20 minutes telephone support biweekly (Masson et al. 2013), mail correspondence twice a week (ter Huurne et al. 2015), and weekly mail contact + automated feedback from the program (Carrard et al. 2011) and six brief biweekly meetings (Grilo \& Masheb 2005). First we 
416 present the results on all eight studies, followed by the results on the four studies that provided adequate 417 and reasonable dose of support (Carrard et al. 2011; Grilo \& Masheb 2005; Masson et al. 2013; ter 418 Huurne et al. 2015).

419 Remission was presented in six studies $(n=333)$ (Carrard et al. 2011; Carter \& Fairburn 1998; 420 Grilo \& Masheb 2005; Grilo et al. 2014; Masson et al. 2013; Shapiro et al. 2007) and the result was in 421 favor of self-help compared with wait list, $\mathrm{RD}=0.25$. Binge eating frequency was reported in seven 422 studies $(n=358)$ (Carrard et al. 2011; Carter \& Fairburn 1998; Grilo \& Masheb 2005; Grilo et al. 2014; 423 Kelly \& Carter 2014; Masson et al. 2013; Shapiro et al. 2007) and the result was in favor of self-help vs. 424 wait list at end of treatment, $\mathrm{SMD}=-0.51$. Eating disorder psychopathology was reported with the EDE425 Q in six studies $(n=348)$ (Carrard et al. 2011; Carter \& Fairburn 1998; Grilo et al. 2014; Kelly \& Carter 426 2014; Masson et al. 2013) and the result was in favor of self-help, $\mathrm{MD}=-0.58$. BMI was reported in 427 seven studies $(n=384)$ (Carrard et al. 2011; Carter \& Fairburn 1998; Grilo \& Masheb 2005; Grilo et al. 428 2014; Kelly \& Carter 2014; Shapiro et al. 2007; ter Huurne et al. 2015) and the result showed no 429 significant difference between self-help vs. wait list. Symptoms of depression was reported in five studies 430 ( $n=282)$ (Carrard et al. 2011; Grilo \& Masheb 2005; Grilo et al. 2014; Kelly \& Carter 2014; ter Huurne 431 et al. 2015) and the result was in favor of the self-help compared with wait list (Table 1), $\mathrm{SMD}=-0.31$, 432 albeit a small and clinically not a meaningful effect. Remission and binge eating frequency was presented in three studies ( $n=192)$ (Carrard et al. 2011; Grilo \& Masheb 2005; Masson et al. 2013) and the result was in favor of self-help compared with wait list, $\mathrm{RD}=0.32[95 \% \mathrm{CI}: 0.20 ; 0.43]$ and $\mathrm{SMD}=-0.56$ [95\% CI:-0.86; -0.26) respectively. Eating disorder psychopathology was reported with the EDE-Q in three studies $(n=219)$ (Carrard et al. 2011; Masson et al. 2013; ter Huurne et al. 2015) and the result was in favor of self-help, MD $=-0.56$ [95\% CI: $-0.85 ;-0.28] . B M I$ was reported in three studies $(n=220)$ (Carrard et al. 2011; Grilo \& Masheb 2005; ter

441 Huurne et al. 2015) and the result showed no significant difference between self-help vs. wait list, $\mathrm{MD}=$ - 
4420.73 [95\% CI:-3.93;2.48]. Symptoms of depression was reported in three studies $(n=211)$ (Carrard et al.

443 2011; Grilo \& Masheb 2005; ter Huurne et al. 2015) and the result was in favor of the CBT-gsh compared 444 with wait list, $\mathrm{SMD}=-0.35$ [95\% CI:-0.63; -0.07], albeit a small and clinically not a meaningful effect.

445 Two studies reported follow-ups, one at two months (Shapiro et al. 2007), and at one year (Grilo 446 et al. 2014). There is insufficient evidence for long-term evaluation.

$447 \quad$ Side effects were not reported in any of the studies.

448

449

450

451

452

453

454

455

456

457

458

459

460

461

462

463

464

465

466

467

\section{IPT vs. CBT}

Two studies investigated IPT vs. CBT or CBT-gsh (Wilfley et al. 2002; Wilson et al. 2010) with long term follow ups (Hilbert et al. 2012; Hilbert et al. 2015). Participants were 18 years and older, mean ages were 45 and 49 years, the majority were women (79\% and $83 \%$ ), and with a BMI range of 27 to 48 (Wilfley et al. 2002) and up to 45 (Wilson et al. 2010), with a mean BMI of 36 and 37, respectively. Treatments were 20 weekly group sessions plus 3 individual sessions for IPT and CBT (Wilfley et al. 2002) and the other study had 19 individual sessions of IPT vs. CBT-gsh with ten 25-minute sessions over 24 weeks (Wilson et al. 2010). Both studies reported 12 month follow up data that was included in meta analyses while longer term follow up showed too high rate of drop out in one study ( $>50 \%$ ) (Wilfley et al. 2002).

Remission was reported in both studies at end of treatment $(n=265)$, and at 12 months follow-up $(n=265)$. The treatments were equivalent at end of treatment, and at 12 months follow up (Table 1).

Binge eating frequency was reported in both studies at end of treatment $(\mathrm{n}=299)$ and at 12 months follow up $(n=279)$. Both treatments were equal at both occasions. Eating disorder psychopathology was only reported in one study (Wilson et al. 2010), hence no meta-analyses was performed. BMI was reported in both studies at end of treatment $(n=299)$ and at 12 months follow up $(n=279)$. IPT and CBT were equal at both occasions. Symptoms of depression were only reported in one study (Wilfley et al. 2002) and thus there was insufficient evidence for a meta-analysis.

Side effects were not reported in any of the studies. 

women (63-89\%) with a BMI $\geq 27$. One study included participants with a BMI between 30 and 55 (Grilo et al. 2011) [19]. Two studies compared the effect of BWL and CBT, both in group format, over 16 sessions during 16 weeks (Munsch et al. 2007) and 24 weeks (Grilo et al. 2011). One study investigated 24 weeks of BWL (20 sessions) with CBT-gsh including 10 sessions of 25 minutes long support (Wilson et al. 2010). One study compared CBT-gsh vs. BWL-gsh with biweekly 20-minute sessions during 12 weeks (Grilo \& Masheb 2005). Three studies investigated long-term follow-ups (Grilo et al. 2011; Munsch et al. 2007; Wilson et al. 2010) Remission was reported in all four studies at end of treatment $(n=375)$ and by three studies at 1-year follow up $(n=300)$ (Grilo et al. 2011; Munsch et al. 2007; Wilson et al. 2010). At end of treatment there was no significant difference between BWL and CBT (group/CBT-gsh), but at 1 year follow up there was a significant difference in favor of CBT (group/CBT-gsh) compared with BWL, RD=-0.13. studies at 1 year follow up $(n=300)$ (Grilo et al. 2011; Munsch et al. 2007; Wilson et al. 2010)]. At end of treatment and at 1 year follow up there were significant difference in favor of the CBT conditions, $\mathrm{SMD}=0.27$, and $\mathrm{SMD}=0.24$ respectively. Eating disorder psychopathology was only reported in one study (Wilson et al. 2010). BMI was reported in all four studies at end of treatment $(n=376)$ and in three studies at the 1-year follow up $(n=300)$ (Grilo et al. 2011; Munsch et al. 2007; Wilson et al. 2010). We found no significant difference between BWL and CBT at any of the time points. Symptoms of depression were reported in three studies at end of treatment $(n=222)$ (Grilo \& Masheb 2005; Grilo et al. 2011;

491 Munsch et al. 2007), and in two studies at 1-year follow up ( $n=133$ ) (Grilo et al. 2011; Munsch et al. 492 2007). We did not find any significant differences between BWL and CBT at end of treatment or at the 1493 year follow up. 


\section{Discussion}

497

The aim of this systematic review was to evaluate the efficacy and the quality of evidence of psychological, pharmaceutical and combined treatments for BED, and potential side effects. We found moderate-quality evidence for remission (i.e., discontinuation of binge eating) for the comparisons CBT versus wait list and CBT-gsh/self help (sh) vs. wait list. CBT was associated with 400 more per 1000 in remission and CBT-gsh/sh 250 more per 1000 compared with wait list. Moderate-quality evidence was also found for reduction of eating disorder specific psychopathology for CBT-gsh/sh vs. wait list. The results above had the moderate degree of confidence in this systematic review. The IPT and CBT comparisons had low-quality evidence for remission, BE frequency, and weight loss. However, they were equally efficacious at end of treatment and at one-year follow-up for these outcomes. the long-term effect of pharmacotherapy is largely unknown. Interestingly, the effect of SSRIs on depressed mood in BED was not significant despite SSRIs being anti-depressant medications.

510 Lisdexamfetamine was the only intervention showing some positive effect on BMI, a finding that is

511 expected and encouraging, as partial loss of appetite is a known side effect of this category of drugs.

513 Nervous System (CNS) stimulants, and SSRI. The effects of IPT were similar to those of CBT, although

514 the quality of the evidence in that regard was low. However, it should be noted that psychological

515 treatments in most studies have been compared to a wait list control group while pharmaceutical

516 treatments are generally compared to a placebo condition. Given the difference in the nature of the control

517 group in psychological versus pharmaceutical studies, and lack of head-to-head studies, any indirect

518 comparisons should be interpreted with caution. 
519 Available recent systematic reviews of treatment of DSM-IV/DSM-5 BED published in the last decade

520 were scrutinized with focus on RCTs (please see the Introduction). Overall, the general findings were

521 similar across reviews, but there is currently no data to allow evaluation of longer-term effects of

522 pharmacotherapy-only treatment for BED. In terms of limitations, the majority of reviews mention the

523 inconsistencies of outcomes measures across trials, the lack of long-term follow-ups, especially in trials

524 on drug treatment of BED, and methodological shortcomings that lead to exclusion of a significant

525 number of trials from systematic reviews and meta-analyses.

526 The present review and meta-analyses confirms most of the conclusions from previous reviews.

527 The majority of studies did not report long-term follow-up. Lack of information in pharmaceutical studies

528 about the outcome and use or discontinued use of drugs after post-treatment was striking. For CBT, CBT-

529 gsh, IPT, and BWL, follow-ups were available up to one year after the end of the treatment in a few

530 studies with low risk of bias. However, even fewer studies reported longer outcome than one year post

531 treatment, and usually with low quality and focusing on very few variables. Importantly, we observed

532 lack of data on the specific psychopathology of BED and quality of life, even though patients with BED

533 are known to have reduced quality of life. Only two studies included a measure of quality of life (Golay et

534 al. 2005; Ricca et al. 2010). This is an important area for future research, together with health economic

535 studies, which are also lacking.

536 Despite a fair total number of studies investigating the efficacy of treatments for BED in general,

537 not many studies met the fairly generous inclusion criteria and survived the check upon the few exclusion

538 criteria to be included in the current systematic review. In addition, this review clearly shows that a

539 mentionable number of studies have relatively small samples, thus imposing limitation to reach at robust

540 pooled data to investigate some of the specific research questions of interest. As an example, we found

541 too few studies investigating the efficacy of several drugs such as NDRI and SNRI, and psychological

542 treatments such as schema therapy to run meta-analyses. In some cases, such as for studies on

543 anorexiants, we found only two studies that could not be merged due to lack of adequate data. Attempts to

544 retrieve necessary data for our analyses from the authors was met with no response. 
546 were weighted by withdrawing points from the overall rating of these studies. Lack of clarity in some

547 aspects of the procedure (e.g., details of randomization) in several studies resulted in adjustment of the

548 quality rating of such studies. As an example, in some studies the allocation of participants was not

549 described adequately (i.e., to provide transparency) or, in some studies, not at all. In other studies, it was

550 unclear to what extent the assessors were blinded to the allocation of the participants at post-treatment or

551 at follow-up, which resulted in adjustment of the quality rating. Another indication of good quality of the

552 included studies is use of structured or semi-structured interviews for establishing the diagnosis of BED.

553 The exclusion criteria in the studies that were included into the current meta-analyses were logical and

554 reflective of everyday clinical praxis (e.g., excluding those with psychosis or acute suicidality to make

555 sure they receive adequate treatment), and thus not contributing to create limitation, or bias in terms of

556 generalizability of the outcome of the current meta-analysis.

557 The majority of the participants were adult females with BED and with concurrent overweight or

558 obesity, recruited via ads, or in some studies through referrals from different clinics. It is noteworthy that

559 we found no studies that included adolescents. The extent to which the conclusions of the current review

560 can be generalized to other populations (men, adolescents, or minority groups) is limited. However, the

561 severity of the symptoms, and the comorbid psychopathology of the included participants indicate that the

562 included total sample in the current review is not markedly different from those seeking treatment from

563 specialist psychiatry and other relevant caregivers. In all the included studies, participants suffer from full

564 or sub-threshold BED based on the DSM-IV (American Psychiatric Association 1994), DSM-IV-TR

565 (American Psychiatric Association 2000), or other equivalent diagnostic systems. The majority of those

566 with a sub-threshold BED diagnosis according the above mentioned diagnostic manuals would meet the

567 full BED diagnosis according to the most recent version of the DSM (i.e., DSM-5: American Psychiatric

568 Association 2013) given the changes made in DSM-5 with regard to duration and frequency of binge

569 eating episodes. 
571 treatments, but in the majority of the included studies, a clinically adequate dose of intervention was

572 delivered. Titration and optimization of pharmaceutical treatments seem to become more common in

573 newer studies, making them ecologically more valid. The length of most of the psychological treatments

574 varied between 10 and 24 weeks, but a few studies had considerably shorter duration (e.g., a self-help

575 treatment that was only three weeks long). The treatment content among the included psychological

576 treatment studies varied substantially as well. As several essential components of CBT for ED were

577 missing in some treatment packages in several trials, they could not be combined with standard CBT

578 treatment in other studies in a meaningful way, and were thus not entered into any meta-analysis. This led

579 to inclusion of only four comparisons between CBT and wait list.

580 Compliance is an important factor in the interpretation of outcome of treatment trials. For

581 pharmaceutical trials, compliance was fairly high, but in some of the important multicenter studies, the

582 compliance was not higher than $50 \%$ due to side effects of medication, early drop-out, divergence from

583 the trial protocol, lack of effects, or other reasons. Improved procedures in pharmaceutical studies such as

584 titration and regular checks increase the safety of the participants, transparency, and fidelity. Lack of

585 compliance was easier to detect in newer well-controlled studies, compared to earlier studies. For

586 psychological treatments, compliance was reported in some of the studies by defining good compliance in

587 terms of attending a specific number of sessions in face-to-face treatments, or completing a specific

588 number of modules in self-help treatments, while other studies did not report a clear picture of

589 compliance. In studies where compliance was reported, it ranged between 60-93 percent, and although no

590 specific and statistically robust and reliable pattern emerged, compliance seemed to be higher in face-to-

591 face treatments compared to guided self-help treatments.

592 The drop-out rate in the studies included in this meta-analysis was acceptable as a direct

593 consequence of the inclusion and exclusion criteria of the current study. Treatment studies with high rate

594 of drop-out (i.e., $>30 \%$ ) were deemed too biased to be included in the meta-analysis. We also noted a

595 tendency toward using more advanced and adequate statistical analysis (e.g., multilevel modeling) in 
596 more recent studies that more efficiently handle the drop-out and provide intention to treat analysis per

597 default. Half of the studies investigating psychological treatments reported and investigated the drop-out

598 in light of demographic factors and outcome variables. Lower socio-economic status and higher severity

599 of symptoms seemed to be related to higher drop-out, but it was not systematically investigated in the

600 current study.

601 Two other interesting observations were made during the analyses. The first one was lack of an a

602 priori power analysis in a marked number of included studies, especially the older studies. The second

603 one was vague and general statements regarding conflict of interest in some of the pharmaceutical studies

604 with regard to the relationship between those that conducted the study and the pharmaceutical companies

605 supplying the drugs.

606 With respect to pharmacological treatments, the adverse effects associated with SSRI and

607 lisdexamfetamine when described for other disorders (Frampton 2016; Kostev et al. 2014) were also

608 present for participants with BED. The risk for adverse events from psychological treatments is largely

609 unknown; the included studies did not report any adverse events but it is unclear whether this was

610 systematically investigated. In future studies, systematic screening and report of adverse effects should be

611 part of psychological treatment trials as well.

612 In terms of the clinical implications, our findings would add support to the current guidelines

613 such as NICE (National Institutet for Health and Care Excellence 2017) and Royal Australian and New

614 Zealand College of Psychiatrists clinical practice guidelines for the treatment of eating disorders (Hay et

615 al. 2014). A recent review of nine evidence-based clinical guidelines (Hilbert et al. 2017) showed that

616 current guidelines endorse the main empirically supported treatments with considerable agreement, but

617 they differ markedly in additional recommendations. The NICE guidelines do not suggest use of

618 pharmacotherapy as the sole treatment of BED. Lack of research on the long-term efficacy of for example

619 SSRI or lisdexamfetamine in our review adds support to the recommendation of NICE when it comes to

620 pharmacotherapy. CBT-based treatments (in guided self-help-, group-, or individual format) are suggested

621 as the first line treatment of choice. IPT might be employed if CBT is not producing effects, or if the 
622 patient does not wish to receive CBT. This recommendation is also in line with our findings, indicating

623 limited scientific evidence of IPT.

624 Several limitations of the current meta-analysis and review should be mentioned to provide a

625 framework for adequate interpretations. In the planning phase of the present study, we discussed running

626 separate analysis for treatment of BED with, and without obesity. It turned out that the majority of

627 participants in the studies had significant overweight or obesity. The number of studies meeting the

628 criteria for the present meta-analysis was too limited to allow separate analysis with regard to weight

629 status. Access to raw data from several studies and data aggregation might provide a very valuable source

630 for investigating the outcome of different treatments for BED in relation to weight status of the

631 participants. Choice of inclusion and exclusion variables and how they are defined on a scale from

632 conservative to liberal does affect the outcome of any review and meta-analysis. Although our criteria

633 might be considered to belong to the more conservative side, we believe that clear-cut and fairly

634 conservative criteria are necessary to identify studies with sound methodology to make valid inferences.

635 This is, however, a pre-analytic assumption, and the choice and definition of criteria for inclusion and

636 exclusion can always be discussed. This may per se justify the need for replication of not only original

637 studies, but also reviews and meta-analyses. Including studies with high risk of bias may introduce too

638 much uncertainty to make significant gains from including a larger number of studies in a review. As an

639 example, studies with high level of attrition might produce highly biased outcomes that are systematically

640 related to the pattern of attrition. Empirical investigation of a liberal versus a conservative approach in

641 systematic reviews might be highly informative for future decisions about the choice of inclusion and

642 exclusion criteria.

643 In conclusion, we found moderate support for the efficacy of CBT and CBT-gsh (with moderate

644 quality of evidence), and modest support for IPT, SSRI and lisdexamfetamine (with low quality of

645 evidence) in the treatment of adults with BED in terms of cessation of or reduction in the frequency of

646 binge eating. It should be noted that males and adolescents were underrepresented in the included studies.

647 Lisdexamfetamine was the only treatment that showed a clinically non-significant and very modest effect 
648

649

650

651

652

653

654

655

656

657

658

659

660

661

662

663

664

665

666

667

668

669

670

671

672

673

674

675

676

677

678

679

680

681

682

683

684

on weight loss (with low quality of evidence). While there is limited support for the long-term effect of psychological treatments, we have currently no data to ascertain the long-term effect of drug treatments. Pharmaceutical treatments were coupled with some undesired side effects compared to placebo, but the side effects of psychological treatments are unknown. Direct comparisons between pharmaceutical and psychological treatments are needed as well as data to investigate the generalizability of these results to adolescents. Long-term follow-ups, standardized assessments including measures of quality of life, and the study of underrepresented populations should be a priority for future research.

\section{Acknowledgements}

As this is a systematic review, and not an original study, no ethical approval or consent to participate was obtained. As no details, images, or videos relating to any individual are included, no consent for publication was obtained.

\section{References}

Agh T, Kovacs G, Pawaskar M, Supina D, Inotai A, and Voko Z. 2015. Epidemiology, health-related quality of life and economic burden of binge eating disorder: a systematic literature review. Eat Weight Disord 20:1-12.

Agras WS, Telch CF, Arnow B, Eldredge K, Wilfley DE, Raeburn SD, Henderson J, and Marnell M. 1994. Weight loss, cognitive-behavioral, and desipramine treatments in binge eating disorder: An additive design. Behav Ther 25:225-238.

American Psychiatric Association. 1994. Diagnostic and statistical manual of mental disorders. Washington, D. C.: American Psychiatric Press, Inc.

American Psychiatric Association. 2000. Diagnostic and statistical manual of mental disorders (4th ed., text rev.). Washington, D.C.: Author.

American Psychiatric Association. 2013. Diagnostic and statistical manual of mental disorders Washington, DC: Authors.

Amianto F, Ottone L, Abbate Daga G, and Fassino S. 2015. Binge-eating disorder diagnosis and treatment: a recap in front of DSM-5. BMC Psychiatry 15:70. 10.1186/s12888-015-0445-6

Brownley KA, Berkman ND, Peat CM, Lohr KN, Cullen KE, Bann CM, and Bulik CM. 2016. BingeEating Disorder in Adults: A Systematic Review and Meta-analysis. Ann Intern Med 165:409420. 10.7326/M15-2455

Brownley KA, Peat CM, La Via M, and Bulik CM. 2015. Pharmacological approaches to the management of binge eating disorder. Drugs 75:9-32. 10.1007/s40265-014-0327-0

Brownley KA, Von Holle A, Hamer RM, La Via M, and Bulik CM. 2013. A double-blind, randomized pilot trial of chromium picolinate for binge eating disorder: results of the Binge Eating and Chromium (BEACh) study. J Psychosom Res 75:36-42. 
685

686

687

688

689

690

691

692

693

694

695

696

697

698

699

700

701

702

703

704

705

706

707

708

709

710

711

712

713

714

715

716

717

718

719

720

721

722

723

724

725

726

727

728

729

730

731

732

733

734

735
Carrard I, Crépin C, Rouget P, Lam T, Golay A, and Linden M. 2011. Randomised controlled trial of a guided self-help treatment on the Internet for binge eating disorder. Behaviour research and therapy 49:482-491.

Carter JC, and Fairburn CG. 1998. Cognitive-behavioral self-help for binge eating disorder: a controlled effectiveness study. Journal of consulting and clinical psychology 66:616-623.

Cassin SE, von Ranson KM, Heng K, Brar J, and Wojtowicz AE. 2008. Adapted motivational interviewing for women with binge eating disorder: a randomized controlled trial. Psychol Addict Behav 22:417-425.

Castelnuovo G, Manzoni GM, Villa V, Cesa GL, and Molinari E. 2011. Brief Strategic Therapy vs Cognitive Behavioral Therapy for the Inpatient and Telephone-Based Outpatient Treatment of Binge Eating Disorder: The STRATOB Randomized Controlled Clinical Trial. Clin Pract Epidemiol Ment Health 7:29-37.

Citrome L. 2017. Binge-Eating Disorder and Comorbid Conditions: Differential Diagnosis and Implications for Treatment. J Clin Psychiatry 78 Suppl 1:9-13. 10.4088/JCP.sh16003su1c.02

Claudino AM, de Oliveira IR, Appolinario JC, Cordas TA, Duchesne M, Sichieri R, and Bacaltchuk J. 2007. Double-blind, randomized, placebo-controlled trial of topiramate plus cognitive-behavior therapy in binge-eating disorder. J Clin Psychiatry 68:1324-1332.

Cohen J. 1988. Statistical Power Analysis for the behavioral sciences. New York: Routledge.

Colles SL, Dixon JB, and O'Brien PE. 2008. Grazing and loss of control related to eating: two high-risk factors following bariatric surgery. Obesity (Silver Spring) 16:615-622. 10.1038/oby.2007.101

Corwin RL, Boan J, Peters KF, and Ulbrecht JS. 2012. Baclofen reduces binge eating in a double-blind, placebo-controlled, crossover study. Behav Pharmacol 23:616-625.

de Man Lapidoth J, Ghaderi A, and Norring C. 2006. Eating disorders and disordered eating among patients seeking non-surgical weight-loss treatment in Sweden. Eat Behav 7:15-26. 10.1016/j.eatbeh.2005.05.006

de Man Lapidoth J, Ghaderi A, and Norring C. 2011. Binge eating in surgical weight-loss treatments. Long-term associations with weight loss, health related quality of life (HRQL), and psychopathology. Eat Weight Disord 16:e263-269.

de Zwaan M. 2001. Binge eating disorder and obesity. Int J Obes Relat Metab Disord 25 Suppl 1:S51-55. 10.1038/sj.ijo.0801699

de Zwaan M, Hilbert A, Swan-Kremeier L, Simonich H, Lancaster K, Howell LM, Monson T, Crosby RD, and Mitchell JE. 2010. Comprehensive interview assessment of eating behavior 18-35 months after gastric bypass surgery for morbid obesity. Surg Obes Relat Dis 6:79-85. 10.1016/j.soard.2009.08.011

Decaluwe V, and Braet C. 2003. Prevalence of binge-eating disorder in obese children and adolescents seeking weight-loss treatment. Int J Obes Relat Metab Disord 27:404-409. 10.1038/sj.ijo.0802233

Dingemans AE, Spinhoven P, and van Furth EF. 2007. Predictors and mediators of treatment outcome in patients with binge eating disorder. Behav Res Ther 45:2551-2562.

Fairburn CG, Bailey-Straebler S, Basden S, Doll HA, Jones R, Murphy R, O'Connor ME, and Cooper Z. 2015. A transdiagnostic comparison of enhanced cognitive behaviour therapy (CBT-E) and interpersonal psychotherapy in the treatment of eating disorders. Behav Res Ther 70:64-71. 10.1016/j.brat.2015.04.010

Favaro A, and Santonastaso P. 1997. Suicidality in eating disorders: clinical and psychological correlates. Acta Psychiatr Scand 95:508-514.

Frampton JE. 2016. Lisdexamfetamine: A Review in ADHD in Adults. CNS Drugs 30:343-354. 10.1007/s40263-016-0327-6

Golay A, Laurent-Jaccard A, Habicht F, Gachoud JP, Chabloz M, Kammer A, and Schutz Y. 2005. Effect of orlistat in obese patients with binge eating disorder. Obes Res 13:1701-1708.

Grilo C. 2016. Randomized controlled trial testing behavioral weight loss versus multi-modal steppedcare treatment for binge eating disorder. European psychiatry 33:S163. 
736

737

738

739

740

741

742

743

744

745

746

747

748

749

750

751

752

753

754

755

756

757

758

759

760

761

762

763

764

765

766

767

768

769

770

771

772

773

774

775

776

777

778

779

780

781

782

783

784

785

786

Grilo CM. 2017. Psychological and Behavioral Treatments for Binge-Eating Disorder. J Clin Psychiatry 78 Suppl 1:20-24. 10.4088/JCP.sh16003su1c.04

Grilo CM, Crosby RD, Wilson GT, and Masheb RM. 2012. 12-month follow-up of fluoxetine and cognitive behavioral therapy for binge eating disorder. Journal of consulting and clinical psychology 80:1108-1113.

Grilo CM, and Masheb RM. 2005. A randomized controlled comparison of guided self-help cognitive behavioral therapy and behavioral weight loss for binge eating disorder. Behav Res Ther 43:15091525 .

Grilo CM, Masheb RM, and Salant SL. 2005a. Cognitive behavioral therapy guided self-help and orlistat for the treatment of binge eating disorder: a randomized, double-blind, placebo-controlled trial. Biol Psychiatry 57:1193-1201.

Grilo CM, Masheb RM, White MA, Gueorguieva R, Barnes RD, Walsh BT, McKenzie KC, Genao I, and Garcia R. 2014. Treatment of binge eating disorder in racially and ethnically diverse obese patients in primary care: randomized placebo-controlled clinical trial of self-help and medication. Behav Res Ther 58:1-9.

Grilo CM, Masheb RM, and Wilson GT. 2005b. Efficacy of cognitive behavioral therapy and fluoxetine for the treatment of binge eating disorder: a randomized double-blind placebo-controlled comparison. Biol Psychiatry 57:301-309.

Grilo CM, Masheb RM, Wilson GT, Gueorguieva R, and White MA. 2011. Cognitive-behavioral therapy, behavioral weight loss, and sequential treatment for obese patients with binge-eating disorder: a randomized controlled trial. Journal of consulting and clinical psychology 79:675-685.

Grilo CM, and White MA. 2013. Orlistat with behavioral weight loss for obesity with versus without binge eating disorder: randomized placebo-controlled trial at a community mental health center serving educationally and economically disadvantaged Latino/as. Behav Res Ther 51:167-175.

Grilo CM, White MA, Gueorguieva R, Barnes RD, and Masheb RM. 2013. Self-help for binge eating disorder in primary care: a randomized controlled trial with ethnically and racially diverse obese patients. Behav Res Ther 51:855-861.

Grilo CM, White MA, and Masheb RM. 2009. DSM-IV psychiatric disorder comorbidity and its correlates in binge eating disorder. Int J Eat Disord 42:228-234. 10.1002/eat.20599

Guerdjikova AI, McElroy SL, Kotwal R, Welge JA, Nelson E, Lake K, Alessio DD, Keck PE, Jr., and Hudson JI. 2008. High-dose escitalopram in the treatment of binge-eating disorder with obesity: a placebo-controlled monotherapy trial. Hum Psychopharmacol 23:1-11.

Guerdjikova AI, McElroy SL, Welge JA, Nelson E, Keck PE, and Hudson JI. 2009. Lamotrigine in the treatment of binge-eating disorder with obesity: a randomized, placebo-controlled monotherapy trial. Int Clin Psychopharmacol 24:150-158.

Guerdjikova AI, McElroy SL, Winstanley EL, Nelson EB, Mori N, McCoy J, Keck PE, Jr., and Hudson JI. 2012. Duloxetine in the treatment of binge eating disorder with depressive disorders: a placebo-controlled trial. Int J Eat Disord 45:281-289.

Guerdjikova AI, Mori N, Blom TJ, Keck PE, Jr., Williams SL, Welge JA, and McElroy SL. 2016. Lisdexamfetamine dimesylate in binge eating disorder: a placebo controlled trial. Hum Psychopharmacol 31:382-391.

Guyatt GH, Oxman AD, Vist GE, Kunz R, Falck-Ytter Y, Alonso-Coello P, Schunemann HJ, and Group GW. 2008. GRADE: an emerging consensus on rating quality of evidence and strength of recommendations. BMJ 336:924-926. 10.1136/bmj.39489.470347.AD

Hay P, Chinn D, Forbes D, Madden S, Newton R, Sugenor L, Touyz S, Ward W, Royal A, and New Zealand College of P. 2014. Royal Australian and New Zealand College of Psychiatrists clinical practice guidelines for the treatment of eating disorders. Aust N Z J Psychiatry 48:977-1008. $10.1177 / 0004867414555814$

Hilbert A, Bishop ME, Stein RI, Tanofsky-Kraff M, Swenson AK, Welch RR, and Wilfley DE. 2012. Long-term efficacy of psychological treatments for binge eating disorder. Br J Psychiatry 200:232-237. 
787

788

789

790

791

792

793

794

795

796

797

798

799

800

801

802

803

804

805

806

807

808

809

810

811

812

813

814

815

816

817

818

819

820

821

822

823

824

825

826

827

828

829

830

831

832

833

834

835

836

837

Hilbert A, Hildebrandt T, Agras WS, Wilfley DE, and Wilson GT. 2015. Rapid response in psychological treatments for binge eating disorder. Journal of consulting and clinical psychology 83:649-654.

Hilbert A, Hoek HW, and Schmidt R. 2017. Evidence-based clinical guidelines for eating disorders: international comparison. Curr Opin Psychiatry 30:423-437. 10.1097/YCO.0000000000000360

Hilbert A, and Tuschen-Caffier B. 2004. Body image interventions in cognitive-behavioural therapy of binge-eating disorder: a component analysis. Behav Res Ther 42:1325-1339.

Hoek HW. 2006. Incidence, prevalence and mortality of anorexia nervosa and other eating disorders. Curr Opin Psychiatry 19:389-394. 10.1097/01.yco.0000228759.95237.78

Hudson JI, Hiripi E, Pope HG, Jr., and Kessler RC. 2007. The prevalence and correlates of eating disorders in the National Comorbidity Survey Replication. Biol Psychiatry 61:348-358. 10.1016/j.biopsych.2006.03.040

Hudson JI, McElroy SL, Raymond NC, Crow S, Keck PE, Jr., Carter WP, Mitchell JE, Strakowski SM, Pope HG, Jr., Coleman BS, and Jonas JM. 1998. Fluvoxamine in the treatment of binge-eating disorder: a multicenter placebo-controlled, double-blind trial. Am J Psychiatry 155:1756-1762.

Iacovino JM, Gredysa DM, Altman M, and Wilfley DE. 2012. Psychological treatments for binge eating disorder. Curr Psychiatry Rep 14:432-446. 10.1007/s11920-012-0277-8

Kelly AC, and Carter JC. 2014. Self-compassion training for binge eating disorder: A pilot randomized controlled trial. Psychol Psychother 0.

Kessler RC, Berglund PA, Chiu WT, Deitz AC, Hudson JI, Shahly V, Aguilar-Gaxiola S, Alonso J, Angermeyer MC, Benjet C, Bruffaerts R, de Girolamo G, de Graaf R, Maria Haro J, KovessMasfety V, O'Neill S, Posada-Villa J, Sasu C, Scott K, Viana MC, and Xavier M. 2013. The prevalence and correlates of binge eating disorder in the World Health Organization World Mental Health Surveys. Biol Psychiatry 73:904-914. 10.1016/j.biopsych.2012.11.020

Kostev K, Rex J, Eith T, and Heilmaier C. 2014. Which adverse effects influence the dropout rate in selective serotonin reuptake inhibitor (SSRI) treatment? Results for 50,824 patients. Ger Med Sci 12:Doc15. 10.3205/000200

Kristeller J, Wolever RQ, and Sheets V. 2014. Mindfulness-Based Eating Awareness Training (MB-EAT) for binge eating: A randomized clinical trial. Mindfulness 5:282-297.

Laederach-Hofmann K, Graf C, Horber F, Lippuner K, Lederer S, Michel R, and Schneider M. 1999. Imipramine and diet counseling with psychological support in the treatment of obese binge eaters: a randomized, placebo-controlled double-blind study. International Journal of Eating Disorders 26:231-244.

Leombruni P, Piero A, Lavagnino L, Brustolin A, Campisi S, and Fassino S. 2008. A randomized, double-blind trial comparing sertraline and fluoxetine 6-month treatment in obese patients with Binge Eating Disorder. Prog Neuropsychopharmacol Biol Psychiatry 32:1599-1605.

Masheb RM, Grilo CM, and Rolls BJ. 2011. A randomized controlled trial for obesity and binge eating disorder: low-energy-density dietary counseling and cognitive-behavioral therapy. Behav Res Ther 49:821-829.

Masson PC, von Ranson KM, Wallace LM, and Safer DL. 2013. A randomized wait-list controlled pilot study of dialectical behaviour therapy guided self-help for binge eating disorder. Behav Res Ther 51:723-728.

McElroy SL. 2017. Pharmacologic Treatments for Binge-Eating Disorder. J Clin Psychiatry 78 Suppl 1:14-19. 10.4088/JCP.sh16003su1c.03

McElroy SL, Casuto LS, Nelson EB, Lake KA, Soutullo CA, Keck PE, Jr., and Hudson JI. 2000. Placebocontrolled trial of sertraline in the treatment of binge eating disorder. Am J Psychiatry 157:10041006.

McElroy SL, Guerdjikova AI, Mori N, Blom TJ, Williams S, Casuto LS, and Keck PE, Jr. $2015 \mathrm{a}$. Armodafinil in binge eating disorder: a randomized, placebo-controlled trial. Int Clin Psychopharmacol 30:209-215.

McElroy SL, Guerdjikova AI, Mori N, Munoz MR, and Keck PE. 2015b. Overview of the treatment of binge eating disorder. CNS Spectr 20:546-556. 10.1017/S1092852915000759 
838

839

840

841

842

843

844

845

846

847

848

849

850

851

852

853

854

855

856

857

858

859

860

861

862

863

864

865

866

867

868

869

870

871

872

873

874

875

876

877

878

879

880

881

882

883

884

885

McElroy SL, Guerdjikova AI, Winstanley EL, O'Melia AM, Mori N, Keck Jr PE, and Hudson JI. 2011a. Sodium oxybate in the treatment of binge eating disorder: An open-label, prospective study. International Journal of Eating Disorders 44:262-268.

McElroy SL, Guerdjikova AI, Winstanley EL, O'Melia AM, Mori N, McCoy J, Keck PE, Jr., and Hudson JI. 2011b. Acamprosate in the treatment of binge eating disorder: a placebo-controlled trial. Int $J$ Eat Disord 44:81-90.

McElroy SL, Hudson J, Ferreira-Cornwell MC, Radewonuk J, Whitaker T, and Gasior M. 2015c. Lisdexamfetamine Dimesylate for Adults with Moderate to Severe Binge Eating Disorder: Results of Two Pivotal Phase 3 Randomized Controlled Trials. Neuropsychopharmacology 0.

McElroy SL, Hudson JI, Capece JA, Beyers K, Fisher AC, and Rosenthal NR. 2007. Topiramate for the treatment of binge eating disorder associated with obesity: a placebo-controlled study. Biol Psychiatry 61:1039-1048.

McElroy SL, Hudson JI, Malhotra S, Welge JA, Nelson EB, and Keck PE, Jr. 2003. Citalopram in the treatment of binge-eating disorder: a placebo-controlled trial. J Clin Psychiatry 64:807-813.

McElroy SL, Hudson JI, Mitchell JE, Wilfley D, Ferreira-Cornwell MC, Gao J, Wang J, Whitaker T, Jonas J, and Gasior M. 2015d. Efficacy and safety of lisdexamfetamine for treatment of adults with moderate to severe binge-eating disorder: a randomized clinical trial. JAMA Psychiatry 72:235-246.

McIntosh VVW, Jordan J, Carter JD, Frampton CMA, McKenzie JM, Latner JD, Joyce PR, Author A, Department of Psychological Medicine U, of Otago CNZ, Canterbury, District Health Board CNZ, Department of Psychology UoC, Christchurch NZ, Psychology Department UoHaMH, United S, Correspondence A, and V.V.W. McIntosh DoPMUoO. 2016. Psychotherapy for transdiagnostic binge eating: A randomized controlled trial of cognitive-behavioural therapy, appetite-focused cognitive-behavioural therapy, and schema therapy. Psychiatry Res 240:412420.

Moher D, Liberati A, Tetzlaff J, Altman DG, and Group P. 2009. Preferred reporting items for systematic reviews and meta-analyses: the PRISMA Statement. Open Med 3:e123-130.

Munsch S, Biedert E, Meyer A, Michael T, Schlup B, Tuch A, and Margraf J. 2007. A randomized comparison of cognitive behavioral therapy and behavioral weight loss treatment for overweight individuals with binge eating disorder. Int J Eat Disord 40:102-113.

National Institutet for Health and Care Excellence. 2017. Eating Disorders: Recognition and Treatment. https://www.nice.org.uk/guidance/ng69: National Institute for Health and Care Excellence.

Nicholls DE, Lynn R, and Viner RM. 2011. Childhood eating disorder: British national surveillance

study. British Journal of Psychiatry 198:295-301.

Pearlstein T, Spurell E, Hohlstein LA, Gurney V, Read J, Fuchs C, and Keller MB. 2003. A double-blind, placebo-controlled trial of fluvoxamine in binge eating disorder: a high placebo response. Arch Womens Ment Health 6:147-151.

Peterson CB, Mitchell JE, Crow SJ, Crosby RD, and Wonderlich SA. 2009. The efficacy of self-help group treatment and therapist-led group treatment for binge eating disorder. Am J Psychiatry 166:1347-1354.

Reas DL, and Grilo CM. 2014. Current and emerging drug treatments for binge eating disorder. Expert Opin Emerg Drugs 19:99-142.

Reas DL, and Grilo CM. 2015. Pharmacological treatment of binge eating disorder: update review and synthesis. Expert Opin Pharmacother 16:1463-1478.

Review Manager (RevMan). 2014. The Nordic Cochrane Centre: The Cochrane Collaboration.

Ricca V, Castellini G, Mannucci E, Lo Sauro C, Ravaldi C, Rotella CM, and Faravelli C. 2010. Comparison of individual and group cognitive behavioral therapy for binge eating disorder. A randomized, three-year follow-up study. Appetite 55:656-665. 
886

887

888

889

890

891

892

893

894

895

896

897

898

899

900

901

902

903

904

905

906

907

908

909

910

911

912

913

914

915

916

917

918
Schlup B, Munsch S, Meyer AH, Margraf J, and Wilhelm FH. 2009. The efficacy of a short version of a cognitive-behavioral treatment followed by booster sessions for binge eating disorder. Behav Res Ther 47:628-635.

Shapiro JR, Reba-Harrelson L, Dymek-Valentine M, Woolson SL, Hamer RM, and Bulik CM. 2007. Feasibility and acceptability of CD-ROM-based cognitive-behavioural treatment for binge-eating disorder. Eur Eat Disord Rev 15:175-184.

Stice E, Marti CN, and Rohde P. 2013. Prevalence, incidence, impairment, and course of the proposed DSM-5 eating disorder diagnoses in an 8-year prospective community study of young women. $J$ Abnorm Psychol 122:445-457. 10.1037/a0030679

ter Huurne ED, de Haan HA, Postel MG, van der Palen J, VanDerNagel JE, and DeJong CA. 2015. WebBased Cognitive Behavioral Therapy for Female Patients With Eating Disorders: Randomized Controlled Trial. J Med Internet Res 17:e152.

Ulfvebrand S, Birgegard A, Norring C, Hogdahl L, and von Hausswolff-Juhlin Y. 2015. Psychiatric comorbidity in women and men with eating disorders results from a large clinical database. Psychiatry Res 230:294-299. 10.1016/j.psychres.2015.09.008

Vamado PJ, Williamson DA, Bentz BG, Ryan DH, Rhodes SK, O'Neil PM, Sebastian SB, and Barker SE. 1997. Prevalence of binge eating disorder in obese adults seeking weight loss treatment. Eat Weight Disord 2:117-124.

Vocks S, Tuschen-Caffier B, Pietrowsky R, Rustenbach SJ, Kersting A, and Herpertz S. 2010. Metaanalysis of the effectiveness of psychological and pharmacological treatments for binge eating disorder. Int J Eat Disord 43:205-217.

White MA, and Grilo CM. 2013. Bupropion for overweight women with binge-eating disorder: a randomized, double-blind, placebo-controlled trial. J Clin Psychiatry 74:400-406.

Wilfley DE, Welch RR, Stein RI, Spurrell EB, Cohen LR, Saelens BE, Dounchis JZ, Frank MA, Wiseman CV, and Matt GE. 2002. A randomized comparison of group cognitive-behavioral therapy and group interpersonal psychotherapy for the treatment of overweight individuals with binge-eating disorder. Arch Gen Psychiatry 59:713-721.

Wilfley DE, Wilson GT, and Agras WS. 2003. The clinical significance of binge eating disorder. Int J Eat Disord 34 Suppl:S96-106. 10.1002/eat.10209

Wilson GT. 2011. Treatment of binge eating disorder. Psychiatr Clin North Am 34:773-783.

Wilson GT, Wilfley DE, Agras WS, and Bryson SW. 2010. Psychological treatments of binge eating disorder. Arch Gen Psychiatry 67:94-101. 10.1001/archgenpsychiatry.2009.170 


\section{Figure 1 (on next page)}

Search results and inclusion of the studies 
Peapula J results from Cochrane Library,

Embase, Psycinfo, Pubmed and Scopus, $\mathrm{n}=3595$

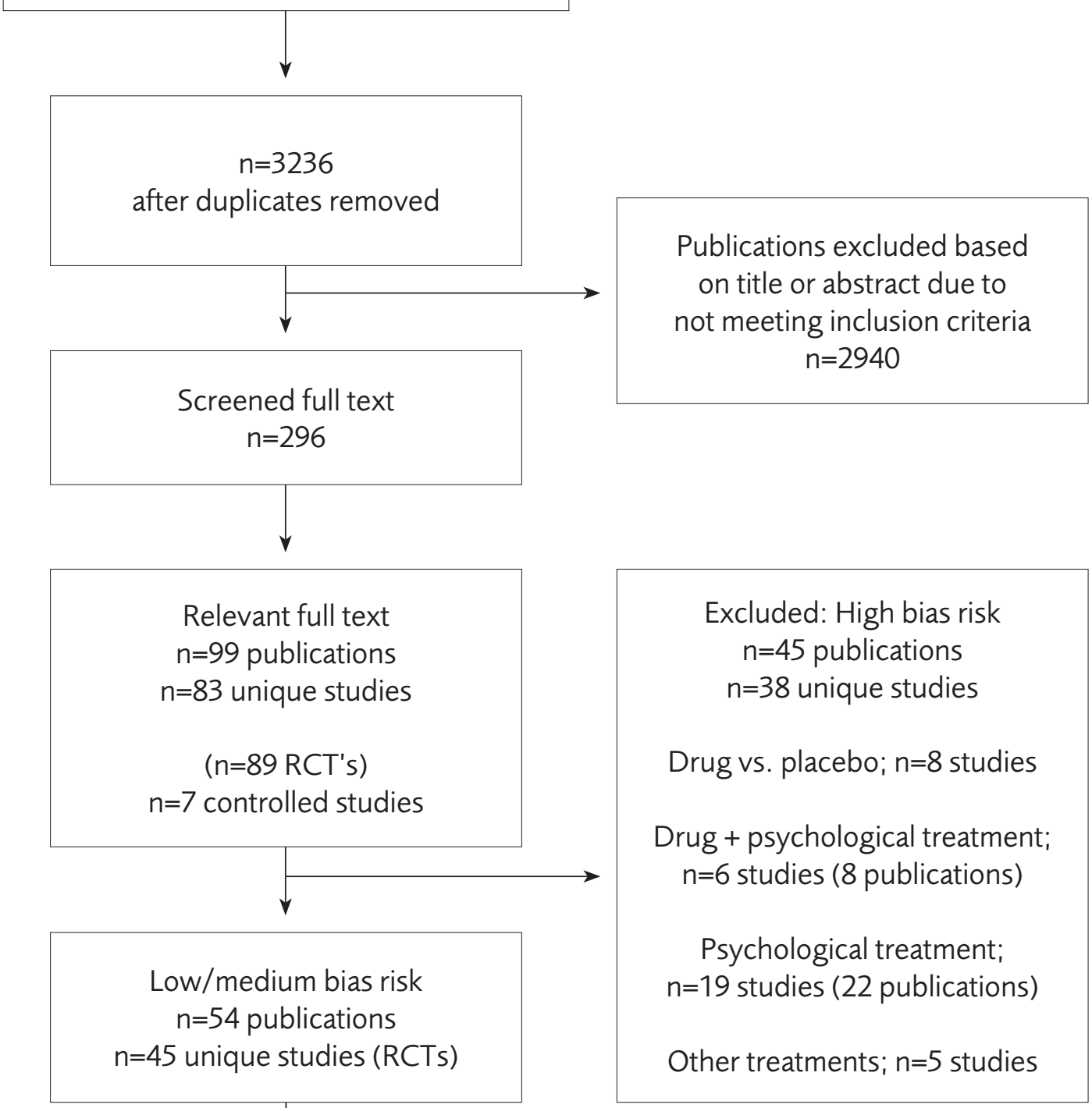

Drug

vs. placebo

Low/medium bias risk $\mathrm{n}=17$ publications

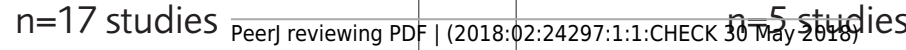

Drug + Psychological Tx

vs. placebo

Low/medium bias risk $\mathrm{n}=7$ publications
Psychological Tx vs. wait-list or other Tx

Low/medium bias risk $\mathrm{n}=30$ publications $\mathrm{n}=23$ studies 


\section{Table $\mathbf{1}$ (on next page)}

Effect size and quality grade of included studies along with the reason for reduction of quality grade.

The number of studies for each comparison, along with number of participants, effect sizes, intervention length and quality of the studies are presented in detail. 


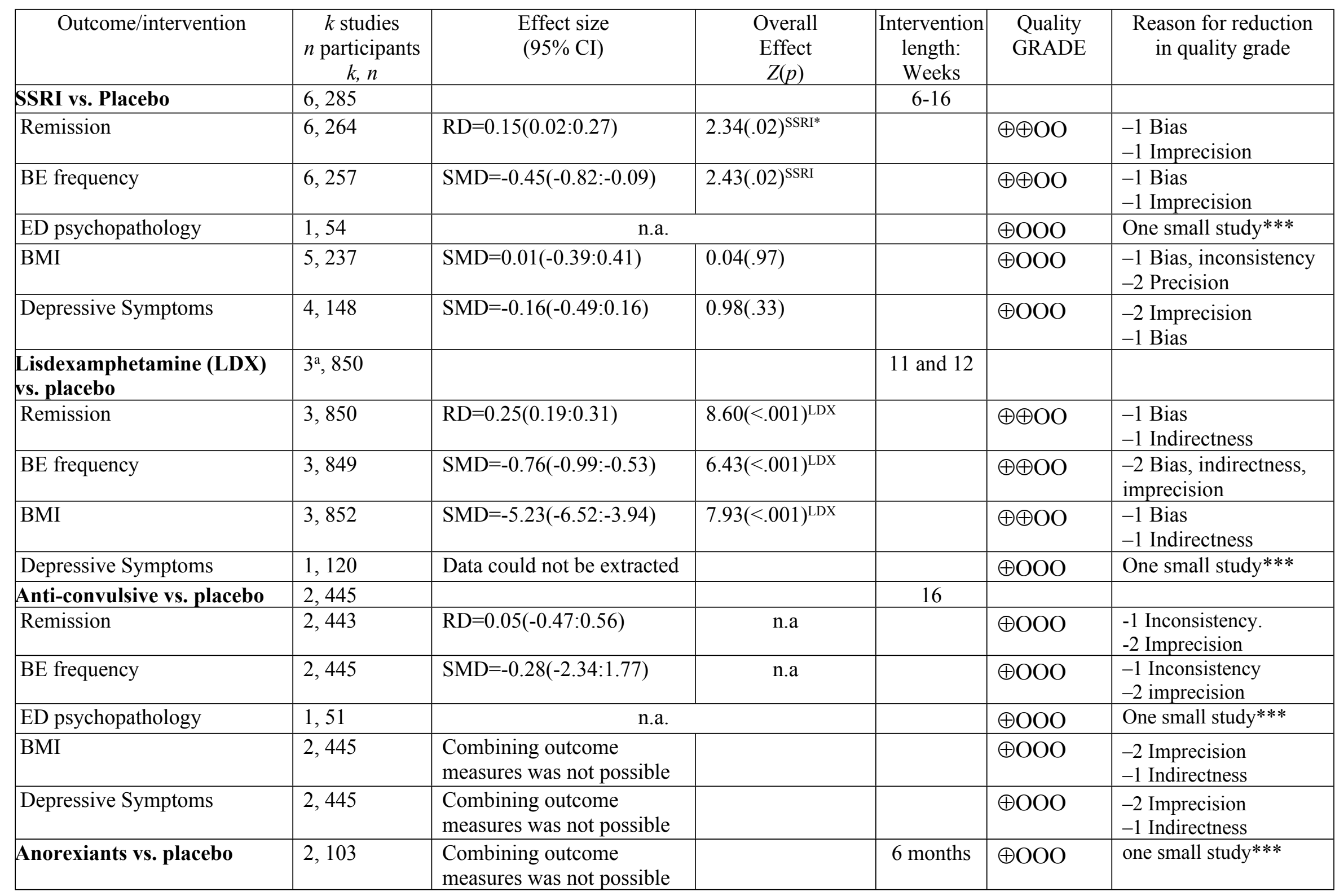




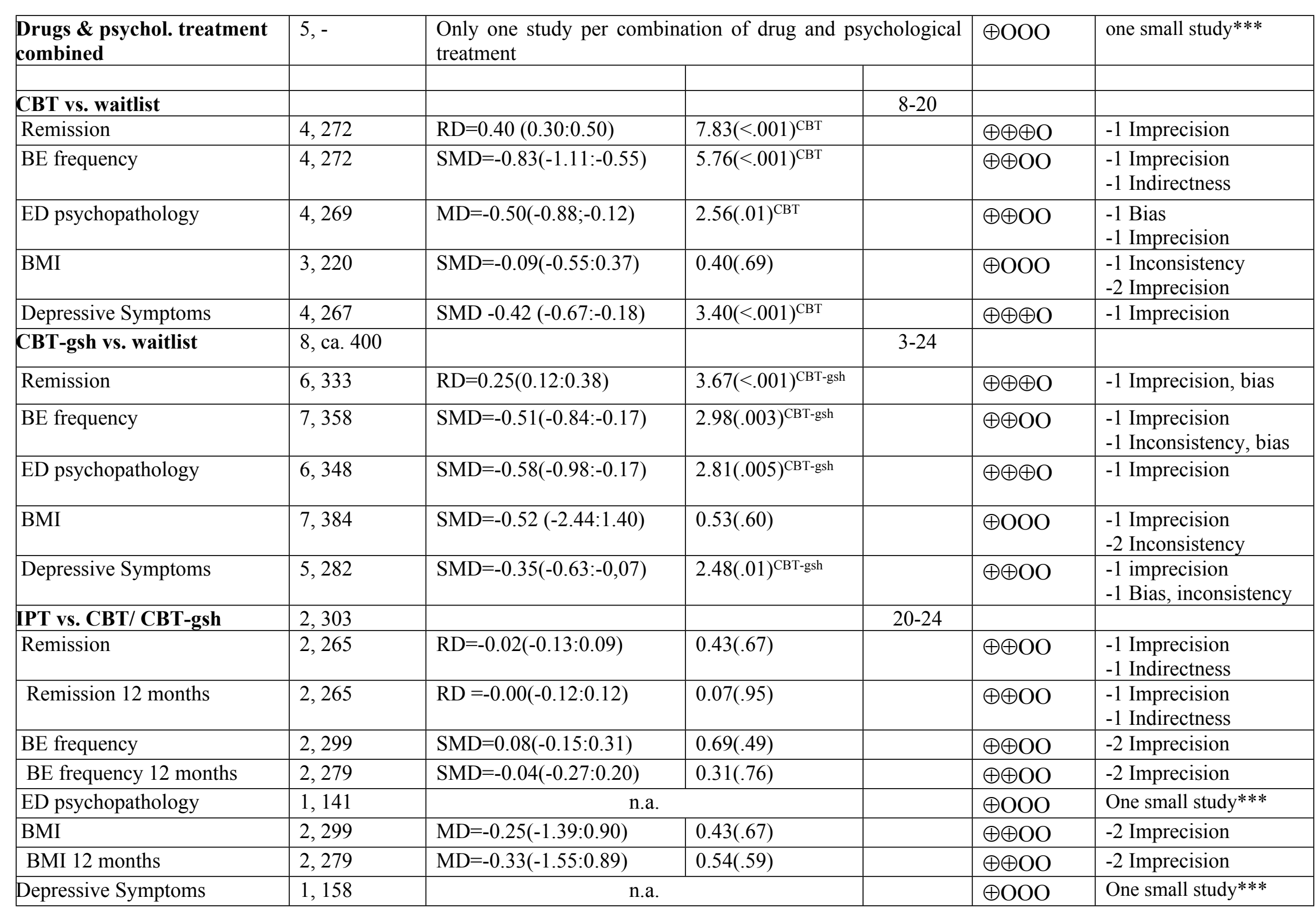




\begin{tabular}{|c|c|c|c|c|c|c|}
\hline BWL vs. CBT ** & 4,375 & & & $12-20$ & & \\
\hline Remission & 4,375 & $\mathrm{RD}=-0.06(-0.22: 0.11)$ & $0.64(.52)$ & & $\oplus \mathrm{OOO}$ & $\begin{array}{l}-1 \text { Inconsistency } \\
-2 \text { Imprecision }\end{array}$ \\
\hline Remission 12 months & 3,300 & $\mathrm{RD}=-0.13(-0.25:-0.02)$ & $2.35(.02)^{\mathrm{CBT}}$ & & $\oplus \oplus \mathrm{OO}$ & -2 Imprecision \\
\hline BE frequency & 4,375 & $\mathrm{SMD}=0.27(0.05: 0.48)$ & $2.40(.02)^{\mathrm{CBT}}$ & & $\oplus \oplus \mathrm{OO}$ & -2 Imprecision \\
\hline BE frequency 12 months & 3,300 & $\mathrm{SMD}=0.24(0.01: 0.46)$ & $2.03(.04)^{\mathrm{CBT}}$ & & $\oplus \oplus \mathrm{OO}$ & -2 Imprecision \\
\hline ED psychopathology & 1,139 & \multicolumn{2}{|c|}{ n.a. } & & $\oplus \mathrm{OOO}$ & \\
\hline BMI & 4,376 & $\mathrm{SMD}=-1.07(-2.40: 0.25)$ & $1.59(.11)$ & & $\oplus \mathrm{OOO}$ & $\begin{array}{l}\text {-2 Inconsistency } \\
-1 \text { Imprecision }\end{array}$ \\
\hline BMI 12 months & 3,300 & $\mathrm{SMD}=-0.23(-1.46: 0.99)$ & $0.37(.71)$ & & $\oplus \oplus \mathrm{OO}$ & $\begin{array}{l}-1 \text { Inconsistency } \\
-1 \text { imprecision }\end{array}$ \\
\hline Depressive Symptoms & 3,222 & $\mathrm{MD}=1.03(-1.20: 3.25)$ & $0.91(.37)$ & & $\oplus \oplus \mathrm{OO}$ & -2 imprecision \\
\hline $\begin{array}{l}\text { Depressive Symptoms } 12 \\
\text { months }\end{array}$ & 2,133 & $\mathrm{MD}=0.25(-2.53: 3.03)$ & $0.18(.86)$ & & $\oplus \mathrm{OOO}$ & $\begin{array}{l}-2 \text { imprecision } \\
-1 \text { indirectness }\end{array}$ \\
\hline
\end{tabular}

$\mathrm{CBT}=$ Cognitive Behavior Therapy; $\mathrm{BE}=$ Binge Eating; $\mathrm{BMI}=$ Body Mass Index; BWL=Behavior Weight Loss; ED=Eating Disorder; gsh=guided self-help; IPT=Interpersonal Psychotherapy; MD = Mean Difference; SMD= Standardized Mean Difference;

$\mathrm{SSRI}=$ Selective Serotonin Reuptake Inhibitor; RD=Risk Difference

Degree of evidence; $\oplus \oplus \oplus \oplus=$ High, $\oplus \oplus \oplus \mathrm{O}=$ Moderate, $\oplus \oplus \mathrm{OO}=$ Low, and $\oplus \mathrm{OOO}=$ Insufficient (Very low).

$\mathrm{a}=$ one publication involved a multicenter study

* superscript indicate the favored treatment

** see manuscript for the different formats involved

*** The confidence in the estimate/evidence is very low $(\oplus \mathrm{OOO})$ when the evidence is based on only one small study (i.e., -2 points

for imprecision and -1 point for indirectness) 